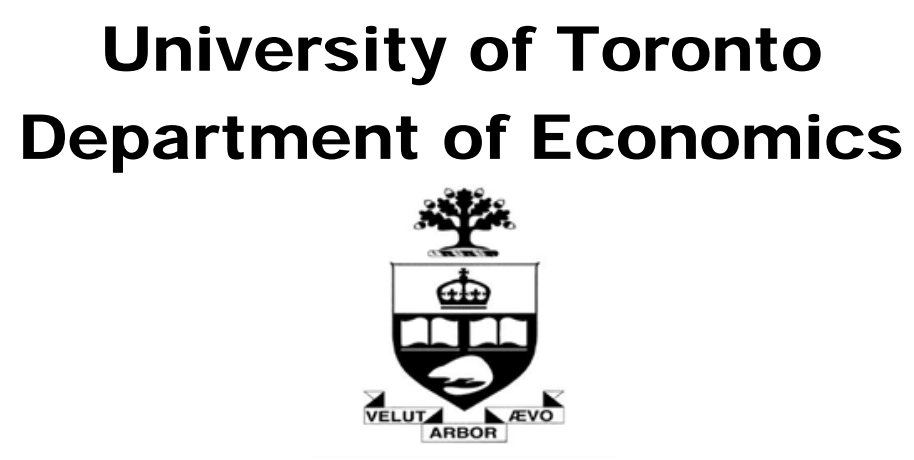

Working Paper 565

Information aggregation with costly reporting

By Martin J. Osborne, Jeffrey S. Rosenthal and Colin Stewart

October 05, 2016 


\title{
Information aggregation with costly reporting
}

\author{
Martin J. Osborne, Jeffrey S. Rosenthal, and Colin Stewart* \\ University of Toronto
}

September 28, 2016

\begin{abstract}
A group of individuals with common interests has to choose a binary option whose desirability depends on an unknown binary state of the world. The individuals independently and privately observe a signal of the state. Each individual chooses whether to reveal her signal, at a cost. We show that if for all revelation choices of the individuals the option chosen by the group is optimal given the signals revealed and the set of individuals who do not reveal signals, then in a large group few signals are revealed, and these signals are extreme. The correct decision is taken with high probability in one state but with probability bounded away from one in the other. No anonymous decision-making mechanism without transfers does better. However, the first-best average payoff can be attained using transfers among agents, and approximately attained with a non-anonymous mechanism without transfers.
\end{abstract}

\section{Introduction}

The job applicant has been grilled, her skills tested, her employment history scrutinized, her references interrogated. Her interviewers have reached various conclusions. Awkwardly polite dinner conversation has led the participants to disparate inferences about her potential devotion to the company's philosophy.

* Osborne and Stewart: Department of Economics; Rosenthal: Department of Statistics. We are grateful to Rahul Deb, Marcin Pęski, Carolyn Pitchik, Matthew A. Turner, and seminar and conference participants for helpful comments on earlier versions of this paper. Osborne thanks Koç University, the Kyoto Institute of Economic Research, and the University of New South Wales for generous hospitality. Rosenthal thanks NSERC and Stewart thanks SSHRC for financial support of this research. Internal hyperlinks: Pieces of notation that are used after not being mentioned for a while are hyperlinked to their definitions; the number in brackets after each item in the references is hyperlinked to the page on which the item is mentioned. 
Now everyone has to decide whether it is worthwhile to explain their assessments to the personnel manager, perhaps in a meeting or perhaps in a private conversation, depending on the company's protocol. Everyone agrees on the quality bar that should be used to determine whether the applicant is hired; they differ only in the "signals" they have received, and the manager's role is merely aggregative. The manager's decision will be based on all the information available, including not only the signals revealed but also the number of employees who do not reveal signals.

Independent scientists have conducted research on the impact of a public policy. Each of them has to decide whether to engage in the costly enterprise of explaining her research to policy-makers, motivated only by the impact of doing so (or not) on the chosen policy; they all agree on the right policy conditional on the unknown facts. An enlightened government bases its decision on the research communicated and its inference about the results of the research that was not communicated.

Each member of a community has absorbed a variety of information about the effect of a local policy. Now each of them has to decide whether to exert the effort necessary to speak at a local council meeting to make her assessment of the evidence known. Everyone agrees on the best policy given the facts. The council will make a decision based on the assessments revealed and the inference drawn from others' choices not to reveal their assessments.

In each case, if the decision-maker cannot commit to a mechanism, what type of information do the agents reveal? How well is information aggregated? If the decision-maker can commit, which mechanism is best? Can welfare be improved relative to the case without commitment?

Model We study a simple model designed to capture the essential features of such situations and answer these questions. Two states of the world are possible, 0 and 1. Each member of a group of $n$ people, whom we call agents, has the same prior belief about the probability of each state and observes the realization of a private signal, a number between 0 and 1 whose distribution depends on the state. Conditional on the state, the realizations of the signals are independent across agents. The probability density $f_{0}$ of the signal in state 0 is decreasing in the signal and the density $f_{1}$ in state 1 is increasing in the signal, as in Figure 1, so that the receipt of a small signal provides relatively strong evidence of state 0 and the receipt of a large one provides relatively strong evidence of state 1. Having observed a signal, each agent decides whether, at the cost $c$, to reveal it. These decisions are made simultaneously. A moderator observes the signals that are revealed and the set of agents who do not reveal signals, and chooses one of two options. Everyone agrees that option 0 is best if the state is 0 and option 1 is best 


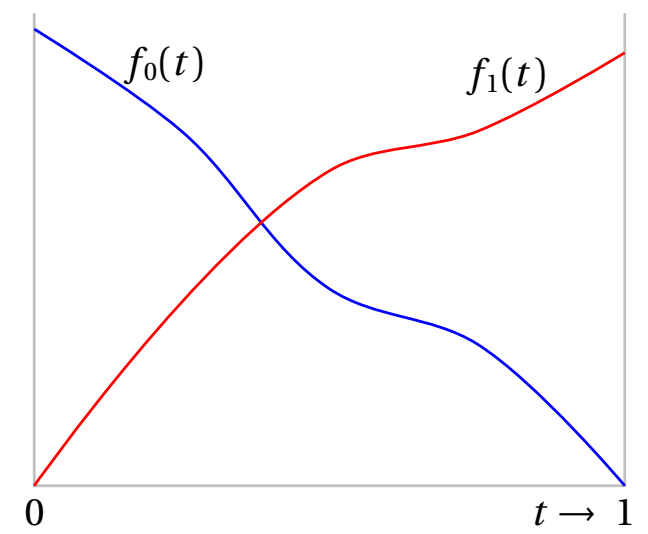

Figure 1. An example of the probability densities of each agent's signal, $t$. In state $s$, the density of each agent's signal is $f_{s}$.

if the state is 1 . The payoff of each agent is 1 if the option matches the state and she does not reveal a signal and $1-c$ if she does; otherwise, her payoff is 0 if she does not reveal a signal and $-c$ if she does. ${ }^{1}$

We start by assuming that the moderator chooses an option that maximizes the common expected payoff given her belief about the state, which depends on her information, namely the signals that are revealed and the set of agents who do not reveal signals. (She makes her decision after the signals are revealed, when the costs of revelation have been borne, so the payoff she maximizes does not include these costs.) Subsequently we ask whether the moderator can do better if she can impose transfers among agents (Section 5.1) or if she can commit to a decision rule that, for some collections of signals, specifies an option that is not optimal (Section 5.2).

Results We restrict attention to symmetric equilibria, in which the set of signals each agent reveals is the same. We show (Proposition 1) that the common strategy of the agents in every such equilibrium has a threshold form: either there is a signal $\underline{x}>0$ such that the set of signals revealed is $[0, \underline{x}]$, or there is a signal $\bar{x}$ such that the set of signals revealed is $[\bar{x}, 1]$, or there are signals $\underline{x}>0$ and $\bar{x}>0$ such that the set of signals revealed is $[0, \underline{x}] \cup[\bar{x}, 1]$. We refer to an equilibrium of the first two types as one-sided and to one of the last type as two-sided.

The structure of the set of equilibria is complex, even with only two agents. Our main results concern properties of the equilibria when the number of agents is large.

\footnotetext{
${ }^{1}$ Note that we do not allow agents to misreport their signals. Given that they have common interests, no agent would have an incentive to misreport if she could; every equilibrium of the game we study would remain an equilibrium if reports were "cheap talk".
} 
In equilibrium, an agent reveals a signal only if doing so improves the moderator's decision enough to compensate for the cost of revelation. When the number of agents is large, some agents are likely to receive extreme, highly informative signals. Thus the revelation of a moderate signal is likely to have little effect on the decision. Consequently, the thresholds in any symmetric equilibrium are close to 0 and 1: only extreme signals are revealed (Lemma 1). Further, the number of agents who reveal signals is bounded, regardless of the size of the group: if many signals are revealed, the improvement in the quality of the decision when another signal is revealed is slight, even if that signal is very informative, and is insufficient to compensate for the cost of revelation (Lemma 3).

When the number of agents is sufficiently large, the game has no two-sided equilibrium. To understand why, consider the possibility of a two-sided equilibrium in which the moderator chooses option 0 if no signal is revealed. Since the equilibrium expected number of agents revealing signals close to 1 is bounded in state 1 , and is much lower in state 0 , it is likely that no such signals occur in state 0 . Thus the moderator is likely to make the correct decision in state 0 even if no signal close to 0 is revealed, implying that revealing low signals is not optimal. This logic is formalized in Proposition 2.

When the number of agents is large, the game has two equilibria, one in which the moderator chooses option 0 if no signal is revealed, and one in which she chooses option 1 in this case. Each agent's ex ante expected payoff is higher in the former equilibrium than in the latter if and only if the prior probability of state 0 exceeds $\frac{1}{2}$. In the former equilibrium, the probability that the moderator chooses the right option is close to 1 when the state is 0 , and close to $1-c$ when the state is 1 (see Proposition 3); information is perfectly aggregated in state 0 , but the costliness of revelation inhibits perfect aggregation in state 1 . If the probability of the moderator's choosing option 1 in state 1 were higher than $1-c$, then for a large enough number of agents, this probability would remain higher than $1-c$ were a given agent to switch from revealing to not revealing her signal, regardless of the signal. Thus her revealing her signal would increase the probability of a correct decision by less than $c$, making revelation of a signal in favor of state 1 suboptimal. We conclude that erroneous decisions must occur with probability at least $c$ in one of the states for agents to reveal signals in favor of that state. Thus for at least one state, $1-c$ is an upper bound-in the limit as the number of agents increases without bound-on the probability of a correct decision in that state.

A similar logic applies to more general mechanisms. Suppose that rather than being bound to choose an option that maximizes the common expected payoff given her belief, the moderator can commit to a decision rule that, for some sets of revealed signals, chooses the suboptimal option. Consider an agent 
who reveals some signals. For her to be willing to do so, if she does not reveal a signal then the moderator must choose the wrong option with probability at least $c$ in at least one state. If she does reveal her signal, her expected payoff is at most $1-c$. Thus, regardless of her action, in at least one state her payoff is at most $1-c$. Hence when the number of agents is large, the ex ante expected payoff of an agent who reveals some signal is at most the larger of her ex ante expected payoffs in the two equilibria of the game in which the moderator does not commit to a decision rule. If the moderator is restricted to anonymous decision rules, in which the selected option depends only on the set of revealed signals, not which agent revealed which signal, this implies that no agent can do better than in the game without commitment, and the likelihood that the moderator makes a correct decision cannot be increased (Corollary 1 ).

In contrast, average payoffs arbitrarily close to 1 can be attained if the moderator can use decision rules that discriminate among the agents, or if the mechanism can include transfers. For example, the moderator can select $k$ agents and commit to choose the optimal option according to their signals if they all reveal signals, and the suboptimal option if any of them does not reveal a signal, ignoring any signals revealed by other agents. If $k$ is large enough, the option chosen by this rule is almost certainly correct if all $k$ agents reveal signals and is almost certainly incorrect otherwise. Thus all $k$ agents optimally reveal their signals and the remaining agents do not. The number $k$ that is sufficiently large is independent of the total number of agents, so for a total number large enough, the agents' average expected payoffs are arbitrarily close to 1.

With transfers, the first-best outcome can be implemented, regardless of the number of agents. We show (Lemma 10) that the first-best strategy profile is an equilibrium of the game in which the revelation cost is $c / n$ (rather than $c$ ) and construct, in Proposition 4, a budget-balanced tax-subsidy scheme that realizes this cost. That is, the scheme taxes those who do not reveal signals and subsidizes those who do so that the net cost of revelation is exactly $c / n$.

For the hiring decision in our first example, the manager's control over the agents' salaries makes transfers possible, so that our results imply that the first-best outcome, with the optimal amount of information revelation, can be achieved.

In the other two examples, in which scientists choose whether to explain their research to policy makers and residents choose whether to attend a meeting about a local policy, transfers appear to be more difficult to implement. Thus our results imply that if we want to treat individuals symmetrically, no advantage is to be gained from committing to a scheme to incentivize scientists to write op-eds on public policy or to one that induces residents to attend local council meetings. Rather, a decision maker should simply act optimally given the signals revealed. 
Related ideas In our model without commitment, an agent's revealing her signal cannot make the outcome worse, because the moderator chooses the best option given the signals revealed. The only reason for an agent not to reveal her signal is the cost of doing so; if this cost is zero, then an agent optimally reveals every signal. If the choice for an agent is not whether to reveal her signal $x$, but rather whether to reveal some coarser information $g(x)$, then her calculation has another ingredient: given what she knows about the process generating the other agents' signals, and hence the signals they are likely to reveal, revealing $g(x)$ may make the outcome worse. Thus if, for example, each agent is limited to casting a vote, as in the model of Feddersen and Pesendorfer $(1996,1997)$, then even if voting is costless she may be better off abstaining if she is poorly informed about the state. The model of this type that comes closest to ours may be that of McMurray (2013), in which the qualities of agents' information are drawn from a continuous distribution, and in equilibrium each agent votes for an option if her posterior belief that the state matches that option is high enough.

In Feddersen and Pesendorfer's model, the fact that voting is costless leads to full information aggregation even though agents can reveal only coarsenings of their signals. This result survives if voting is costly, with each voter's cost drawn independently from a distribution with support $[0, c]$ for some $c>0$ (Krishna and Morgan, 2012).

In these models, the decision rule-majority voting-is fixed. Suppose instead, as we assume initially, that the moderator cannot commit to a decision rule, but rather for any set of revealed signals chooses the best option. Maintain the assumption in the voting models, however, that the moderator observes only a coarsening of the agents' signals, not the signals themselves. Then intuition suggests that if revelation is costless, information will be perfectly aggregated in a large population. Battaglini (2016) studies a variant of such a model in which the moderator's preferences differ from those of the agents (and the agents' actions are interpreted as "protests"). ${ }^{2}$ If the difference between the preferences is large enough, information aggregation is inhibited.

Models in which information is costly to disclose go back at least to Jovanovic (1982) and Verrecchia (1983). More recently, Kartik, Suen, and Xu (2014) study strategic interaction among multiple senders with disclosure costs. This line of work focuses on environments in which senders seek to move the receiver's beliefs in a particular direction, whereas the "senders" in our model always benefit from the receiver's having more information.

Our results that in an equilibrium a small number of extreme signals are revealed are reminiscent of results in an earlier paper with overlapping authorship, Osborne, Rosenthal, and Turner (2000). However, the logic behind the results

\footnotetext{
${ }^{2}$ Lohmann $(1993,1994)$ previously studied a related model of protests.
} 
is completely different. In the earlier paper, information is perfect and agents' preferences differ; the option chosen is the median favorite option of participants. The reason moderate preferences are not expressed in equilibrium is not because they have intrinsically low value. Rather, the fact that the outcome is the median of the participants' favorite options leads to an equilibrium in which moderates do not participate, because only then does each agent's participation have a sufficiently large effect on the outcome to offset the participation cost.

\section{Model}

A set $\{1, \ldots, n\}$ of agents has to choose one of two options, 0 and 1 . The desirability of each option depends on an unknown state, which is either 0 or 1 . Specifically, every agent prefers option 0 in state 0 and option 1 in state 1 . Every agent's prior belief is that the state is 0 with probability $q \in(0,1)$.

Each agent receives a private signal, a number in $[0,1]$, that is correlated with the state. The agents' signals are generated independently; in state $s$, the distribution of each agent's signal is $F_{s}$, with support $[0,1]$ and density $f_{s}$. We assume that $f_{0}$ is continuous and decreasing, $f_{1}$ is continuous and increasing, and $f_{0}(1)=f_{1}(0)=0 .{ }^{3}$ An example of $f_{0}$ and $f_{1}$ is shown in Figure 1 .

Given the agents' common interests, they all benefit from sharing their signals. However, doing so entails a cost. Specifically, each agent who reveals her signal incurs the cost $c>0$. We study a game in which each agent chooses whether to reveal her signal. ${ }^{4}$

To model the group's collective choice of an option, we introduce a moderator, whose preferences and prior belief about the state are the same as those of every agent. The moderator observes the signals revealed by the agents and chooses an option. In doing so, she uses all the information available to her, including the implications of the agents' strategies for the signals that are not revealed.

In summary, we study an extensive game in which the set of players is the set of agents plus the moderator. The game unfolds as follows.

- Chance selects a state: 0 with probability $q, 1$ with probability $1-q$.

- For each state, chance selects a collection of signals, one for each agent. In state $s(0$ or 1$)$, each agent's signal is drawn from the distribution $F_{s}$ independently.

\footnotetext{
${ }^{3}$ We discuss the assumption that $f_{0}(1)=f_{1}(0)=0$ in Section 6 .

${ }^{4}$ As we mentioned in the Introduction (footnote 1 ), no agent would have an incentive to misreport her signal if she could.
} 
- Each agent observes only her own signal.

- Each agent chooses whether to reveal her signal; these decisions are made simultaneously.

- The moderator observes only the signals revealed.

- The moderator chooses one of the two options, 0 and 1.

We assume that the moderator's payoff is 1 if she chooses option 0 when the state is 0 or option 1 when the state is 1 , and is 0 otherwise. Each agent's payoff is equal to the moderator's payoff if she does not reveal her signal and to the moderator's payoff minus $c$ if she does reveal her signal. We argue in Section A.1, in the appendix, that these assumptions about the cardinal payoffs are essentially without loss of generality as long as there are common interests.

We restrict attention to equilibria in pure strategies. (We discuss mixed strategies in Section 6.) A (pure) strategy $\sigma_{i}$ of agent $i$ is a (measurable) function from the set $[0,1]$ of signals to $\{0,1\}$, where $\sigma_{i}(x)$ is the probability that agent $i$ reveals her signal when it is equal to $x$. A (pure) strategy for the moderator is a function that associates an option (0 or 1 ) with each of her information sets, where an information set corresponds to a list $\left(a_{1}, \ldots, a_{n}\right)$ in which $a_{i} \in[0,1] \cup\{\varnothing\}$ for each agent $i$, where $a_{i}=\varnothing$ means that $i$ does not reveal a signal.

Following the standard approach to extensive games with imperfect information, we assume that for each of her information sets, every player behaves optimally, given a belief about the state ("sequential rationality"). We assume also, again following the standard approach, that this belief is, whenever possible, derived using Bayes' Rule ("consistency"). At two types of information set, both belonging to the moderator, Bayes' Rule cannot be applied. At one type, some agent, say $i$, reveals a signal, say $x_{i}$, that her strategy calls for her not to reveal. The only sensible assumption about the moderator's belief at such an information set seems to be that it is the same as the belief she would hold if $i$ 's strategy were to reveal $x_{i}$, and this is the assumption we adopt. At the other type of information set at which Bayes' Rule cannot be applied, some agent does not reveal a signal even though her strategy calls for her to reveal almost all signals. How the moderator should form a belief about the state at such an information set is unclear. As a consequence, we put few restrictions on this belief. Specifically, for any strategy profile $\sigma$ in which $\sigma_{i}$ reveals almost every signal we assume that there is an alternative strategy $\sigma_{i}^{\prime}$ for which the set of signals not revealed has positive measure, and such that at every information set of the moderator at which $i$ does not reveal a signal, the moderator's belief about the state is derived from $\sigma_{i}^{\prime}$. The main restriction that this assumption entails is that, for any given 
strategy profile $\sigma$, the strategy $\sigma_{i}^{\prime}$ is the same for every information set at which $i$ does not reveal a signal; it does not depend on the other agents' actions at the information set. This restriction seems sensible given that the agents make their decisions independently.

Under these assumptions, the game has no equilibrium in which some agent reveals almost all signals. To see why, suppose that agent $i$ employs a strategy $\sigma_{i}$ that reveals almost all signals. If agent $i$ does not reveal a signal, then the moderator forms her belief about the state on the basis of the alternative strategy $\sigma_{i}^{\prime}$. It is straightforward to argue that for some signal $x_{0}$, this belief is the same as the belief the moderator would form were the agent to reveal $x_{0}$. (If, for example, the signals that $\sigma_{i}^{\prime}$ does not reveal constitute an interval, then $x_{0}$ is a member of that interval.) Because revelation is costly and $i$ can affect the option chosen by the moderator only by affecting her belief, revealing $x_{0}$-and, by continuity, signals close to $x_{0}$-is not optimal for the agent. This observation is formalized in Lemma 4 in the appendix.

Given that the game has no equilibrium in which any agent reveals almost all signals, we describe our notion of equilibrium explicitly only for strategy profiles in which no agent reveals almost all signals, and study only equilibria for which the strategy profile has this property. (Doing so significantly reduces the required notation.)

For any strategy $\sigma_{i}$ of agent $i$ and state $s$, define

$$
h\left(\sigma_{i}, s\right)=\int_{0}^{1}\left(1-\sigma_{i}(x)\right) f_{s}(x) d x,
$$

the probability that $i$ does not reveal a signal when the state is $s$. For any $a_{i} \in$ $[0,1] \cup\{\varnothing\}$, strategy $\sigma_{i}$ of agent $i$, and state $s$, define

$$
w\left(a_{i}, \sigma_{i}, s\right)= \begin{cases}f_{s}\left(a_{i}\right) & \text { if } a_{i} \in[0,1] \\ h\left(\sigma_{i}, s\right) & \text { if } a_{i}=\varnothing\end{cases}
$$

For a strategy profile $\sigma$, the probability the moderator's belief assigns to state 0 at the information set $a=\left(a_{1}, \ldots, a_{n}\right)$ is

$$
\beta_{\sigma}(0 \mid a)=\frac{q \prod_{i=1}^{n} w\left(a_{i}, \sigma_{i}, 0\right)}{q \prod_{i=1}^{n} w\left(a_{i}, \sigma_{i}, 0\right)+(1-q) \prod_{i=1}^{n} w\left(a_{i}, \sigma_{i}, 1\right)}
$$

and the probability it assigns to state 1 is $\beta_{\sigma}(1 \mid a)=1-\beta_{\sigma}(0 \mid a)$. (Given our restriction to strategies in which no agent reveals almost all signals, the denominator is positive when $a_{j}=\varnothing$ for some agent $j$, as well as when $a_{j}$ is a signal.) 
Given a strategy profile $\sigma$, we say that the moderator acts optimally if her strategy $\sigma^{m}$ satisfies

$$
\sigma^{m}(a)= \begin{cases}0 & \text { if } \beta_{\sigma}(0 \mid a)>\beta_{\sigma}(1 \mid a) \\ 1 & \text { if } \beta_{\sigma}(1 \mid a)>\beta_{\sigma}(0 \mid a)\end{cases}
$$

Equivalently, she acts optimally if she chooses option 0 whenever

$$
\frac{\beta_{\sigma}(0 \mid a)}{\beta_{\sigma}(1 \mid a)}=\frac{q}{1-q} \prod_{i=1}^{n} \frac{w\left(a_{i}, \sigma_{i}, 0\right)}{w\left(a_{i}, \sigma_{i}, 1\right)}>1
$$

and option 1 whenever this ratio is less than 1.

For any strategy profile $\sigma$ and signal $x_{i}$ of agent $i$, let $u_{i}\left(x_{i} ; \sigma\right)$ be the expected payoff of type $x_{i}$ of agent $i$, excluding the revelation cost, when she reveals her signal. That is, $u_{i}\left(x_{i} ; \sigma\right)$ is the probability that the option chosen by the moderator is equal to the state when $i$ is of type $x_{i}$ and reveals her signal, given the probability distribution over the moderator's information sets implied by the other agents' strategies and the moderator's beliefs at these information sets implied by $\sigma$. Let $u_{i}(\varnothing ; \sigma)$ be the analogous payoff when $i$ does not reveal a signal.

An equilibrium is a strategy profile $\sigma$ for which an agent of type $x_{i}$ reveals her signal if $u_{i}\left(x_{i} ; \sigma\right)-c>u_{i}(\varnothing ; \sigma)$ and does not reveal it if $u_{i}\left(x_{i} ; \sigma\right)-c<u_{i}(\varnothing ; \sigma)$, and the moderator acts optimally. We restrict attention to equilibria in which every agent's strategy is the same; we refer to such strategy profiles as symmetric, although we often omit this qualifier and write simply "equilibrium".

\section{Equilibria}

\subsection{Examples}

One agent The game is not trivial even in the case of a single agent $(n=1)$. Denote by $P(s \mid x)$ the posterior probability of state $s$ given the signal $x \in[0,1]$ :

$$
P(0 \mid x)=\frac{q f_{0}(x)}{q f_{0}(x)+(1-q) f_{1}(x)} \text { and } P(1 \mid x)=\frac{(1-q) f_{1}(x)}{q f_{0}(x)+(1-q) f_{1}(x)} .
$$

If the agent reveals the signal $x$, the moderator's payoff when she chooses option $z$ is $P(z \mid x)$. Thus in any equilibrium the moderator chooses the option $\arg \max _{z \in\{0,1\}} P(z \mid x)$, so that the expected payoff of type $x$ of the agent is $\max _{z \in\{0,1\}} P(z \mid x)-c$ if she reveals her signal, indicated by the orange curve in Figure 2.

First consider the possibility of an equilibrium in which the moderator chooses option 0 if the agent does not reveal a signal. In this case, the expected 


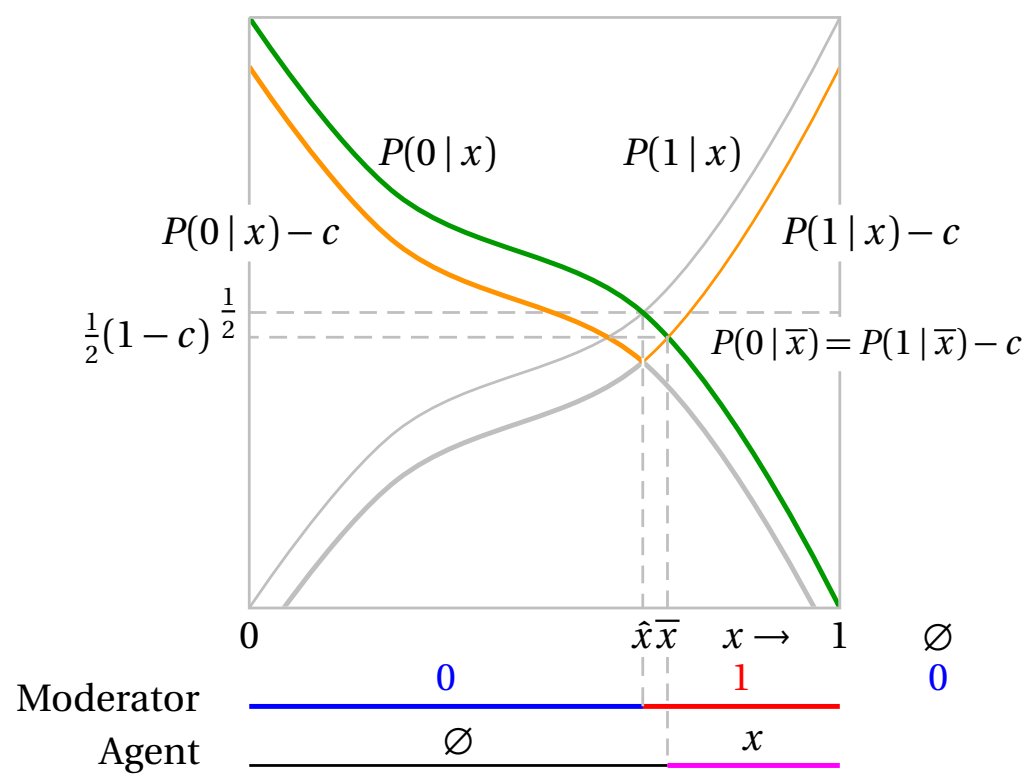

Figure 2. An equilibrium in the case of a single agent. The moderator chooses option 0 if the agent does not reveal a signal or reveals a signal less than $\hat{x}$, and option 1 if she reveals a signal greater than $\hat{x}$. The agent reveals signals greater than $\bar{x}$ and does not reveal signals less than $\bar{x}$.

payoff of type $x$ is $P(0 \mid x)$ if she does not reveal her signal, indicated by the green curve in Figure 2.

Given that $P(0 \mid x)$ is decreasing in $x$ and $P(1 \mid x)$ is increasing in $x$, there exists a signal $\bar{x}$ such that the agent optimally reveals every signal $x>\bar{x}$ and does not reveal any signal $x<\bar{x}$. Specifically, $P(0 \mid \bar{x})=\frac{1}{2}(1-c)$, as illustrated in Figure 2 . The pair of strategies is an equilibrium if, given the agent's strategy, choosing option 0 when the agent does not reveal a signal is optimal for the moderator. The condition for optimality is $\operatorname{Pr}(s=0 \mid x \in[0, \bar{x}]) \geq \frac{1}{2}$, which is true if $q$ is sufficiently large ( $q>\frac{1}{2}$ is enough) or $c$ is sufficiently small.

A symmetric analysis applies to the case in which the moderator chooses option 1 if the agent does not reveal a signal. Thus for small values of $c$ the game has two (pure) equilibria, one in which the agent reveals low signals and one in which she reveals high signals.

Two agents When there are two agents, the structure of the equilibria is considerably more complex, and we do not have a detailed characterization for general signal distributions.

For triangular signal distributions $\left(f_{0}(x)=2(1-x)\right.$ and $\left.f_{1}(x)=2 x\right)$ and $q=\frac{1}{2}$, the equilibria may be calculated explicitly. Equilibria in which the moderator chooses option 0 if no signal is revealed take the following forms. For $c<\frac{3}{2}-\sqrt{2}$, 


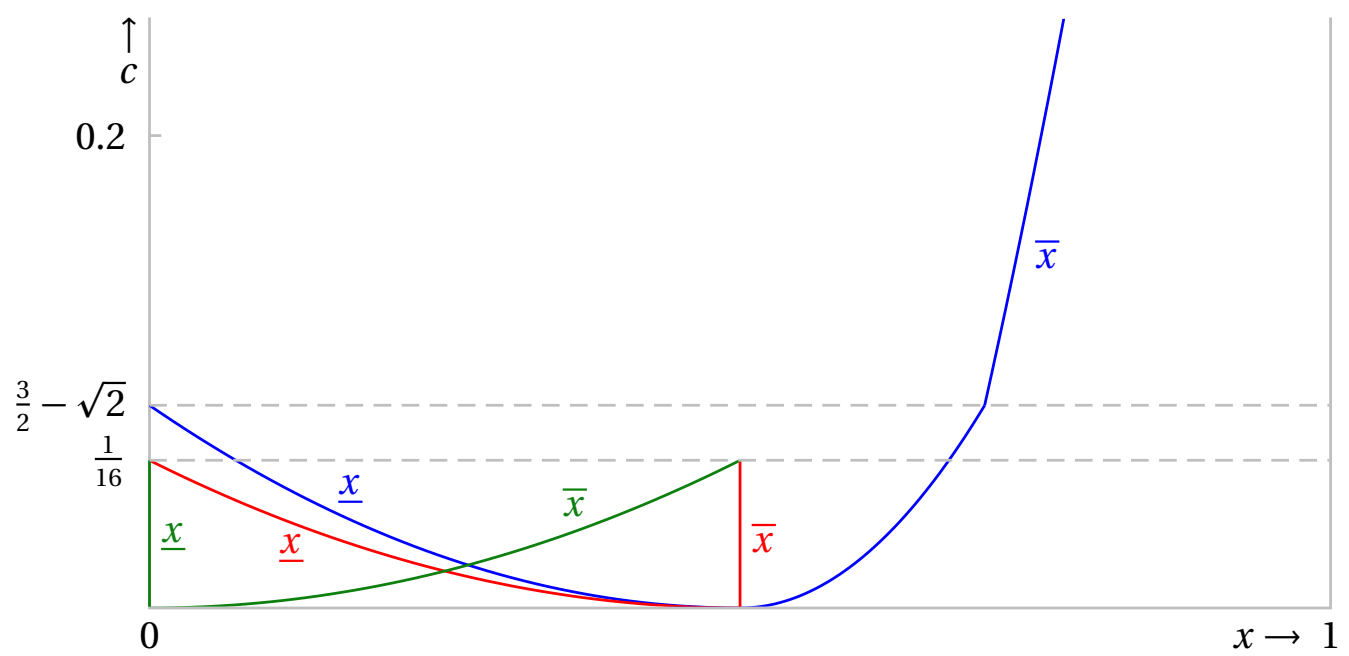

Figure 3. The equilibria when there are two individuals and the moderator chooses option 0 when no signal is revealed, for triangular signal distributions and $q=\frac{1}{2}$. For each value of $c \leq \frac{1}{16}$ there is a continuum of equilibria between the green and red equilibria, with $\bar{x}-\underline{x}=2 \sqrt{c}$.

the game has also an equilibrium in which each agent reveals signals outside $\left[\frac{1}{2}-\left(1+\frac{1}{2} \sqrt{2}\right) \sqrt{c}, \frac{1}{2}+\frac{1}{2} \sqrt{2} \sqrt{c}\right]$ and does not reveal signals in the interior of this interval, and for $c>\frac{3}{2}-\sqrt{2}$ an equilibrium in which each agent reveals signals greater than $\frac{1}{3}(1+\sqrt{1+3 c})$ and does not reveal smaller signals (the blue equilibrium in Figure 3). In this equilibrium, whenever exactly one agent reveals her signal, the moderator chooses the option favored by that signal. If both agents reveal signals, the moderator chooses the option favored by the stronger signal. At the $\bar{x}$ threshold the incentive for an agent to reveal her signal is driven by the possibility that the other agent may not reveal her signal, whereas at the $\underline{x}$ threshold this incentive is driven by the possibility that the other agent will reveal a signal that is close to the threshold but less than $1-\underline{x}$.

In addition, for $c \leq \frac{1}{16}$ for each $\underline{x} \in\left[0, \frac{1}{2}-2 \sqrt{c}\right]$ the game has an equilibrium in which each agent reveals signals outside $[\underline{x}, \underline{x}+2 \sqrt{c}]$ and does not reveal signals in the interior of this interval. ${ }^{5}$ Note that in each of these equilibria an agent reveals the signal $\frac{1}{2}$ even though it is completely uninformative about the state. She does so because if she did not then the moderator would believe that her signal favored state 0 and would choose option 0 even if the other agent revealed a signal moderately in favor of state 1 (although option 1 is best given these signals).

For each of these equilibria there is an equilibrium in which the roles of options 0 and 1 are reversed, and the moderator chooses option 1 if no signal is revealed.

\footnotetext{
${ }^{5}$ This continuum of equilibria collapses to the equilibria with $\underline{x} \approx 0$ and $\underline{x} \approx \frac{1}{2} \sqrt{c}$ for $q$ slightly different from $\frac{1}{2}$.
} 
For the same triangular signal distributions and values of $q$ other than $\frac{1}{2}$ analytic expressions for the equilibrium thresholds seem not to exist. Numerical computations for specific values of $c$ reveal multiple isolated equilibria that depend on the value of $q$ nonmonotonically in ways that are difficult to interpret.

\subsection{Structure of equilibria}

Let $\sigma$ be a symmetric strategy profile, let $a_{i} \in[0,1] \cup\{\varnothing\}$, let $s$ be a state, and let $z$ be an option. Denote by $\psi\left(z, a_{i}, s ; \sigma\right)$ the probability that the moderator chooses option $z$ when the state is $s$, the moderator and $n-1$ agents follow the strategy profile $\sigma$, and the remaining agent's action is $a_{i}$. The actions of the $n-1$ agents who follow $\sigma$ depend on the signals they receive, so the option chosen by the moderator is random even though $\sigma$ is pure.

In any (symmetric) equilibrium, each agent reveals only signals that are sufficiently extreme, and the boundaries between revealing and not revealing a signal are characterized by indifference conditions. To state this result, we define a threshold strategy with thresholds $\underline{x}$ and $\bar{x}$, where $\underline{x} \in[0,1], \bar{x} \in[0,1]$, and $\underline{x}<\bar{x}$, to be a strategy of an agent that reveals every signal in $[0, \underline{x}) \cup(\bar{x}, 1]$ and no signal in $(\underline{x}, \bar{x})$. Note that if $\underline{x}=0$ then $[0, \underline{x})$ is empty and if $\bar{x}=1$ then $(\bar{x}, 1]$ is empty.

Recall that for every strategy of an agent (for which, as we assume throughout, there is a positive measure of signals that the strategy does not reveal), some signal $x_{0}$ generates the same belief for the moderator about the state as does nonrevelation. (See Lemma 4 in the appendix for details.) The last claim in our first result is that in an equilibrium $x_{0}$ is in the interval of signals not revealed. Recall that $P(s \mid x)$ is the posterior probability of state $s$ given the signal $x$ (see (2)).

Proposition 1 In every (symmetric) equilibrium $\sigma$, the agents' common strategy is a threshold strategy. For an equilibrium strategy with thresholds $\underline{x}$ and $\bar{x}$, if $\underline{x} \in$ $(0,1)$ then an agent with signal $\underline{x}$ is indifferent between revealing and not revealing her signal, so that

$$
c=P(0 \mid \underline{x})(\psi(0, \underline{x}, 0 ; \sigma)-\psi(0, \varnothing, 0 ; \sigma))+P(1 \mid \underline{x})(\psi(1, \underline{x}, 1 ; \sigma)-\psi(1, \varnothing, 1 ; \sigma)),
$$

and if $\bar{x} \in(0,1)$ then an agent with signal $\bar{x}$ is indifferent between revealing and not revealing her signal, so that

$$
c=P(0 \mid \bar{x})(\psi(0, \bar{x}, 0 ; \sigma)-\psi(0, \varnothing, 0 ; \sigma))+P(1 \mid \bar{x})(\psi(1, \bar{x}, 1 ; \sigma)-\psi(1, \varnothing, 1 ; \sigma)) .
$$

The value of $x_{0}$ given by Lemma 4 for the agents' common equilibrium strategy is in $(\underline{x}, \bar{x})$. 
Proofs are in Section A.2, in the appendix.

The key idea behind Proposition 1 is that the incentive to reveal a signal is stronger for a signal farther from $x_{0}$. For example, consider signals $x_{i}$ and $x_{i}^{\prime}$ for some agent $i$ with $x_{i}<x_{i}^{\prime}<x_{0}$. The difference between $x_{i}$ and $x_{i}^{\prime}$ affects $i$ 's incentive to reveal her signal in two ways. First, the signal she receives determines her interim belief about the state: she assigns higher probability to state 0 if she receives signal $x_{i}$ than if she receives $x_{i}^{\prime}$. Second, revealing the signal $x_{i}$ leads to a larger increase in the moderator's belief in state 0 , relative to nonrevelation, than does revealing $x_{i}^{\prime}$. To compare the incentives to reveal these two signals, the proof of Proposition 1 compares each to a hypothetical intermediate case in which the agent's belief is based on $x_{i}$ whereas the moderator acts according to $x_{i}^{\prime}$ if the signal is revealed. Since $i$ 's revealing either signal makes the moderator more likely to choose action 0 , the incentive to reveal is stronger in this case than if the agent receives signal $x_{i}^{\prime}$. Since the agent and the moderator have common interests, the agent's incentive to reveal $x_{i}$ is stronger if the moderator acts according to $x_{i}$ than if she acts according to $x_{i}^{\prime}$. Combining these two comparisons, we conclude that the agent has a stronger incentive to reveal signals farther from $x_{0}$.

\section{Large number of agents}

While the structure of the set of equilibria for a fixed number $n$ of agents appears to be complicated (except for $n=1$ ), it becomes relatively simple as $n$ grows large. We start by showing that as $n$ grows large, the thresholds in any equilibrium become extreme. The logic is that if they did not become extreme, then eventually the moderator would have enough information to make the right decision with high probability even if an agent deviated to not revealing her signal; consequently, for no signal would revelation be optimal.

Lemma 1 For every $\varepsilon>0$ there exists a number $N$ such that, for every $n>N$, for any equilibrium of the game with $n$ agents we have $\underline{x}<\varepsilon$ and $\bar{x}>1-\varepsilon$, where $\underline{x}$ and $\bar{x}$ are the thresholds of the common equilibrium strategy.

How accurate are the moderator's decisions? The following result states that in an equilibrium in which agents with signals close to 0 reveal them, the quality of decision-making in state 0 is not too high, and symmetrically for signals close to 1 . Specifically, for $x=0$ or $x=1$, in an equilibrium in which agents reveal signals close to $x$, the probability that the moderator chooses option $x$ in state $x$ can be only slightly higher than $1-c$ when the number of agents is large. Moreover, the bound is uniform across equilibria and across values of $n$ (provided that they are sufficiently large). 
Lemma 2 For every $\varepsilon>0$ there exists a number $N$ such that, for any $n>N$, in any equilibrium of the game with $n$ agents, if $\underline{x}>0$ then the probability that the moderator chooses option 0 in state 0 is at most $1-c+\varepsilon$, and if $\bar{x}<1$ then the probability that the moderator chooses option 1 in state 1 is at most $1-c+\varepsilon$, where $\underline{x}$ and $\bar{x}$ are the thresholds of the common equilibrium strategy.

Roughly, if the moderator is too likely to choose the right option, paying the cost of revealing a signal is not worthwhile for an agent. This rough intuition does not take into account the fact that when an agent deviates from revealing a signal to not revealing it, the option chosen by the moderator may change. Indeed, for an agent with an extreme signal, such a deviation could significantly reduce the probability that the moderator chooses the correct option. However, when averaged across all signals, the effect of such a deviation is small when the number of agents is large: in a symmetric equilibrium, every agent's ex ante impact is the same, and is hence small. In particular, if the equilibrium probability of a correct decision in state $s$ is greater than $1-c$, then for a large enough number of agents, it remains above $1-c$ if a single agent deviates to not revealing any signal. But then if an agent gets an extreme signal in favor of state $s$, she does not want to reveal it because the probability that the moderator chooses option $s$ in state $s$ if the agent does not reveal her signal is already greater than $1-c$.

We now establish that the expected number of agents who reveal signals in an equilibrium remains bounded as the number of agents increases. The basic idea behind this result is the same as for Lemma 1: if the expected number of agents who reveal signals were to increase without bound as the number of agents increases, then eventually the quality of the moderator's decision would be too good to justify any agent's revealing her signal.

Lemma 3 The expected number of agents who reveal signals in equilibrium remains bounded as the number of agents increases without bound.

We say that an equilibrium in which $\underline{x}>0$ and $\bar{x}<1$ is two-sided and one in which either $\underline{x}=0$ and $\bar{x}<1$, or $\underline{x}>0$ and $\bar{x}=1$, is one-sided.

Our next result says that when the number of agents is large the game has no two-sided equilibrium. Suppose that the moderator chooses option 0 if no signal is revealed. By Lemma 3, in state 1 the expected number of agents with signals above $\bar{x}$ who reveal them remains bounded as the number of agents increases. By Lemma 1, the threshold $\bar{x}$ converges to 1 as the number of agents increases, so that $f_{0}(\bar{x}) / f_{1}(\bar{x})$ converges to zero. Thus in state 0 the expected number of agents with signals above $\bar{x}$ who reveal them goes to zero, and in fact the probability that no signal above $\bar{x}$ is revealed goes to 1 . But then in state 0 the moderator almost certainly chooses option 0 regardless of the revealed signals less than $\underline{x}$, 
so that an agent with such a signal has no incentive to reveal it. Hence a twosided equilibrium is not possible.

The next result shows also that when the number of agents is large, the moderator's decision takes a particularly simple form: whenever at least one agent reveals a signal in equilibrium, the moderator takes the opposite decision to the one she takes when no signal is revealed.

Given that $f_{1}(0)=f_{0}(1)=0$ and that $f_{0}$ is decreasing and $f_{1}$ is increasing, for any signal $x \in(0,1)$ and any value of $n$ there is a unique signal $z_{1}(x, n)$ such that the posterior probability of state 0 is $\frac{1}{2}$ given the information that there are $n-1$ signals in $[0, x]$ and one signal equal to $z_{1}(x, n)$. Precisely, $z_{1}(x, n)$ satisfies

$$
\frac{1}{2}=\frac{q F_{0}(x)^{n-1} f_{0}\left(z_{1}(x, n)\right)}{q F_{0}(x)^{n-1} f_{0}\left(z_{1}(x, n)\right)+(1-q) F_{1}(x)^{n-1} f_{1}\left(z_{1}(x, n)\right)}
$$

or, equivalently,

$$
q f_{0}\left(z_{1}(x, n)\right) F_{0}(x)^{n-1}=(1-q) f_{1}\left(z_{1}(x, n)\right) F_{1}(x)^{n-1} .
$$

Proposition 2 There exists a number $N$ such that if $n>N$ then (i) the game has no two-sided equilibrium and (ii) in any one-sided equilibrium in which $\underline{x}=0$ we have $\bar{x} \geq z_{1}(\bar{x}, n)$, where $\underline{x}$ and $\bar{x}$ are the thresholds of the common equilibrium strategy, so that the moderator chooses option 1 if and only if at least one signal (of at least $\bar{x}$ ) is revealed. A symmetric result holds for one-sided equilibria with $\bar{x}=1$.

An implication of this result is that when the number of agents is large, in a one-sided equilibrium in which $\underline{x}=0$ the moderator chooses option 0 if every agent's signal is less than $\bar{x}$ and option 1 if some agent's signal is greater than $\bar{x}$. Thus the probability of a correct decision in such an equilibrium is $F_{0}(\bar{x})^{n}$ in state 0 and $1-F_{1}(\bar{x})^{n}$ in state 1 , so that the ex ante probability of a correct decision is

$$
q F_{0}(\bar{x})^{n}+(1-q)\left(1-F_{1}(\bar{x})^{n}\right)
$$

and an agent's expected payoff is

$$
q F_{0}(\bar{x})^{n}+(1-q)\left(1-F_{1}(\bar{x})^{n}\right)-c\left[q\left(1-F_{0}(\bar{x})\right)+(1-q)\left(1-F_{1}(\bar{x})\right)\right] .
$$

We can now identify the quality of information aggregation in the limit as the number of agents increases without bound.

Proposition 3 In a one-sided equilibrium in which the upper threshold $\bar{x}$ of the common equilibrium strategy is less than 1 , as the number of agents increases the probability of the moderator's choosing option 0 in state 0 approaches 1 , the probability of her choosing option 1 in state 1 approaches $1-c$, and the expected number of agents who reveal signals approaches $-(1-q) \ln c$. 
By Lemma 2, in any equilibrium, for one state the limit of the probability that the moderator chooses the correct option as the number of agents increases without bound is at most $1-c$. Proposition 3 indicates that this bound is achieved in the limit as $n$ grows large: the correct option is chosen with probability $1-c$ in one state and with probability 1 in the other. Thus the limit of the highest expected payoff of any agent in any equilibrium as the number of agents increases without bound is

$$
\bar{\pi}=\max \{q, 1-q\}+(1-c) \min \{q, 1-q\} .
$$

\section{Alternative mechanisms}

Proposition 3 shows that, even for an arbitrarily large number of agents, information aggregation is imperfect and average payoffs are bounded away from the first-best. Can welfare be improved by using a mechanism that differs from the game in which the moderator is bound to choose an optimal option for every set of revealed signals? For example, the mechanism could require the moderator to take a particular action unless at least some fixed number of agents (a "quorum") reveal their signals. Alternatively, the mechanism could specify that the moderator "punish" agents for revealing certain signals by choosing a suboptimal option.

Whether a mechanism exists that improves welfare depends on whether the designer can tax agents and subsidize the cost of revelation. We show (Proposition 4) that, for any number of agents, there exists a budget-balanced transfer scheme under which a strategy profile that maximizes the agents' average payoff is an equilibrium. We show also (Proposition 5) that if transfers are not possible but the moderator can commit to an anonymous decision rule, then the agents' common ex ante expected payoff does not exceed $\bar{\pi}$, defined in (6). That is, no anonymous decision rule for the moderator improves on the limiting equilibrium outcome as the number of agents increases without bound of the game we have studied so far.

\subsection{Mechanisms with transfers}

Suppose the designer can commit to ex post budget-balanced transfers among the agents. That is, the designer chooses a function $t:([0,1] \cup\{\varnothing\})^{n} \rightarrow \mathbb{R}^{n}$ that associates with every list of actions for the agents a list of transfers to the agents such that $\sum_{i=1}^{n} t_{i}\left(a_{1}, \ldots, a_{n}\right)=0$ for every $\left(a_{1}, \ldots, a_{n}\right)$, where $t_{i}$ denotes the $i$ th coordinate of $t$. The game we study is identical to the one described in Section 2 
except that, for each $i$, the transfer $t_{i}$ is added to agent $i$ 's payoff. We refer to any equilibrium of this game as an equilibrium for $t$.

For any given number of agents, the first-best payoff is the maximum average expected payoff for the agents across all strategy profiles.

Proposition 4 Let $t_{i}(a)=(1-k(a) / n) c$ if $i$ reveals a signal and $t_{i}(a)=-k(a) c / n$ otherwise, where $k(a)$ is the number of agents who reveal signals. There exists an equilibrium for $t$ in which the average expected payoff of the agents is equal to the first-best payoff.

This result shows that an appropriately designed transfer scheme generates an efficient outcome. The inefficiency in the equilibria of the game without transfers arises because of an externality: when agent $i$ reveals her signal, all agents, not only agent $i$, benefit. Thus to induce the amount of revelation that maximizes the agents' average payoff, each agent's payoff has to reflect this externality. Because an agent's revelation of her signal benefits all $n$ agents, it is intuitively plausible that the payoffs internalize the externality when each agent's revelation cost is $c / n$ rather than $c$, a result verified in Lemma 10 of the appendix. Accordingly, to prove Proposition 4, it suffices to show that the transfer scheme defined there is budget-balanced and each agent who reveals her signal receives a transfer that is larger by exactly $(n-1) c / n$ than it would have been had she not revealed her signal, making $c / n$ the net revelation cost.

\subsection{Mechanisms without transfers}

Suppose that transfers among the agents are not possible, but the moderator can commit to a strategy, which we refer to as a decision rule, before the agents choose actions. Accordingly, define a decision rule to be a function $d:([0,1] \cup\{\varnothing\})^{n} \rightarrow\{0,1\}$ giving the option chosen for each list of actions of the agents. We study the (possibly asymmetric) Nash equilibria of the (Bayesian) game $G(d)$ among the agents for a fixed decision rule $d$.

The next result shows that for any decision rule $d$ and any equilibrium of $G(d)$, the ex ante expected payoff of any agent who reveals some signals is at most $\bar{\pi}$ (defined by (6)). Agents who reveal no signals may receive higher payoffs, but the result says that any agent who reveals some signals receives no more than her limiting equilibrium expected payoff, as the number of agents increases without bound, in the game in which the moderator always acts optimally.

Proposition 5 For any number of agents, any decision rule d of the moderator, and any equilibrium of the game $G(d)$, the (ex ante) expected payoff of every agent who reveals some signals is at most $\bar{\pi}$. If no type of any agent reveals her signal then 
the expected payoff of every type of every agent is at $\operatorname{most} \max \{q, 1-q\}$, which is at most $\bar{\pi}$.

Here is the idea behind this result. Fix a decision rule and choose an equilibrium and an agent, say $i$, who reveals some signals in the equilibrium. Let $x_{i}$ be a signal she reveals. Then the expected payoff of type $x_{i}$ of agent $i$ if she does not reveal her signal is not more than her expected payoff if she does reveal it, which is at most $1-c$. Thus if agent $i$ does not reveal a signal, in at least one state the probability that the decision rule chooses the correct option is at most $1-c$. This bound limits the ex ante expected payoff of agent $i$ (over all her possible types); it turns out that the upper limit is $\bar{\pi}$.

Now consider a decision rule for which the option selected depends only on the set of signals, not on the identity of the agent who revealed any particular signal. Call such a decision rule anonymous. In a symmetric equilibrium of $G(d)$ for any anonymous decision rule $d$, every agent's expected payoff is the same, so a consequence of Proposition 5 is that in such an equilibrium every agent's expected payoff is no more than $\bar{\pi}$, her limiting expected payoff in the best equilibrium of the game in which the moderator always acts optimally. That is, when the number of agents is large, in a symmetric equilibrium for an anonymous rule, there is no gain to the moderator's being able to commit to a decision rule that selects the suboptimal option under some circumstances.

Corollary 1 For any anonymous decision rule $d$, the expected payoff of every agent in any symmetric equilibrium of $G(d)$ is at most $\bar{\pi}$.

Proof. In any such equilibrium, every agent receives the same expected payoff, so the result follows from Proposition 5.

Proposition 5 does not exclude the possibility of nonanonymous decision rules with equilibria in which the average expected payoff over all agents is greater than $\bar{\pi}$. In fact, when the number of agents is large such decision rules exist in which the average expected payoff is arbitrarily close to 1 . Consider, for example, the following decision rule. Let $k<n$. The decision rule chooses the option that is optimal according to the posterior belief derived from the signals revealed by agents $1, \ldots, k$ if all of these agents reveal a signal, and chooses the option that is suboptimal according to this belief if one or more of these agents does not reveal a signal. (If the posterior belief assigns probability $\frac{1}{2}$ to each state, then the decision rule can choose either option.) In particular, the option chosen by the decision rule is independent of the actions of agents $k+1, \ldots, n$, who consequently do not report their signals. This rule is a variant of a quorum applied to a fixed subset of agents in which the moderator does not ignore revealed 
signals if they are insufficiently numerous, but rather deliberately chooses the wrong option in this case, and ignores any additional signals.

If $k$ is sufficiently large, the option chosen by this decision rule is almost certainly correct if every agent $1, \ldots, k$ reveals her signal and almost certainly incorrect if exactly one of these agents does not reveal her signal. Thus for any value of $c$ less than 1, for $k$ large enough there is an equilibrium for the decision rule in which every type of each agent $1, \ldots, k$ reveals her signal. Hence the probability of a correct decision - which is equal to the equilibrium payoffs of agents $k+1, \ldots, n$-can be made arbitrarily close to 1 by choosing $k$ sufficiently large (and any $n \geq k$ ). How large $k$ needs to be is independent of $n$, so the average expected payoff can be made arbitrarily close to 1 by choosing $k$ sufficiently large and $n$ sufficiently large relative to $k$.

\section{Discussion}

Heterogeneous preferences For simplicity, we have assumed that players have common interests regarding the option chosen. We conjecture that results similar to ours would hold if the players had heterogeneous preferences. For example, suppose that each agent $i$ has a privately known preference type $\zeta_{i} \in \mathbb{R}$ drawn i.i.d. from a bounded interval. Assume that an agent of type $\zeta$ receives a payoff, excluding the revelation cost, of $\zeta$ if the moderator chooses action 0 and the state is $0,2-\zeta$ if the moderator chooses action 1 and the state is 1 , and 0 otherwise. ${ }^{6}$ Note that some types may prefer a particular action regardless of the state (namely, those for which $\zeta \notin[0,1]$ ). As before, an agent incurs a cost of $c$ if she reveals her signal to the moderator, and, to ensure that the problem is nontrivial, the moderator strictly prefers option $s$ in state $s$.

Although preference types vary in their equilibrium thresholds, the forces driving information revelation are essentially the same as in the homogeneous case. Suppose that $\operatorname{Pr}(\zeta>0)>0$ and consider an equilibrium of the extended model in which the moderator chooses option 1 if no agent reveals a signal. For any type to be willing to pay the cost of revealing its signal, doing so must increase its expected payoff by at least $c$. When the number of agents is large, such an increase is possible only for those types most strongly in favor of option 0 those whose signal is close to 0 and whose value of $\zeta$ is high. As in the homogeneous case, if these types obtain a discrete gain from revealing their signals then these signals must be rare in state 1, suggesting that the equilibrium must be one-sided in the sense that no type reveals signals close to 1 . The only difference, then, relative to the homogeneous case, seems to be that the revelation cost

\footnotetext{
${ }^{6}$ This model coincides with our common interest model when $\zeta=1$ almost surely.
} 
must be normalized relative to the gain from changing the moderator's choice. Thus, for example, if $M$ is the supremum of the support of $\zeta$, then we conjecture that, in the limit as the number of agents increases without bound, the moderator almost surely chooses option 1 in state 1 , and chooses option 0 in state 0 with probability $1-c / M$.

Bounded beliefs The assumption that $f_{0}(1)=f_{1}(0)=0$ plays an important role in our analysis. We have verified that our results can be extended to allow $f_{0}(1)$ and $f_{1}(0)$ to be positive as long as they are sufficiently small. However, we do not view the version of our model in which they are nonzero to be very natural. In such a model, there is a hard, commonly known limit on the strength of any individual's belief about the state: the belief of an agent who receives a signal of 1, say, assigns positive probability to both states, but is certain that her belief assigns higher probability to state 1 than does any other agent's. This feature strikes us as undesirable, particularly since there is no such limit on the amount of information collectively available through aggregating individuals' signals. If more precise information may be obtained by the group, why should the precision of any one agent's signal have a firm limit? A formulation that eliminates this undesirable feature seems to require additional correlation in the agents' signals.

Mixed strategies Our analysis is restricted to pure strategies. Allowing agents to mix would have essentially no effect on our results since, in any equilibrium, only the threshold types $\underline{x}$ and $\bar{x}$ are indifferent. Allowing the moderator to mix after no agent reveals a signal could, however, introduce additional equilibria. We focus on pure strategies of the moderator for two reasons. First, we think of the option chosen by the moderator if no agent reveals a signal as a widely understood status quo. The only reasonable nondeterministic choice seems to be the 50-50 mixture of the two options, but that mixture is not in general compatible with an equilibrium. Second, we conjecture that, for a sufficiently large number of agents, there is generically one equilibrium in which the moderator randomizes nontrivially, and that equilibrium is two-sided. By Lemma 2, two-sidedness implies that the correct option is chosen with probability at most $1-c$ in each state (in the limit as the number of agents grows without bound). This equilibrium therefore entails lower welfare than does either of the one-sided pure strategy equilibria. Since we are interested primarily in welfare-maximizing equilibria, it thus makes sense to focus on pure strategies. 


\section{Appendix}

\section{A.1 General payoffs}

We argue that our assumption that the payoffs from the options are 0 and 1 is essentially without loss of generality. First, note that the payoff of 0 for an option that differs from the state is simply a normalization; if the payoff to option $1-s$ in state $s$ is $b \neq 0$, we can subtract $b$ from the payoff to each action in state $s$. Now, taking the payoff to option $1-s$ in state $s$ to be 0 , denote by $b_{s}$ the payoff from option $s$ in state $s$. If $b_{0}$ and $b_{1}$ are both negative, we can relabel the states to make them positive. If one is negative while the other is positive, the moderator has a dominant action and the game is trivial. Accordingly, suppose both are positive. Let $a$ denote the option chosen by the moderator. Given any strategy profile, the expected payoff of any type $x_{i}$ of agent $i$ is

$$
\begin{aligned}
q b_{0} \operatorname{Pr}\left(a=0 \mid s=0, x_{i}\right)+(1-q) b_{1} \operatorname{Pr}\left(a=1 \mid s=1, x_{i}\right)-c \sigma_{i}\left(x_{i}\right) \\
=\left(q b_{0}+(1-q) b_{1}\right)\left[q^{\prime} \operatorname{Pr}\left(a=0 \mid s=0, x_{i}\right)\right. \\
\left.+\left(1-q^{\prime}\right) \operatorname{Pr}\left(a=1 \mid s=1, x_{i}\right)-c^{\prime} \sigma_{i}\left(x_{i}\right)\right],
\end{aligned}
$$

where

$$
q^{\prime}=\frac{q b_{0}}{q b_{0}+(1-q) b_{1}} \quad \text { and } \quad c^{\prime}=\frac{c}{q b_{0}+(1-q) b_{1}} .
$$

Hence the payoffs for type $x_{i}$ are proportional to those in the game with prior $q^{\prime}$, cost $c^{\prime}$, and payoff 1 from action $s$ in each state $s$. The same transformation applies to the moderator. Therefore, a strategy profile is an equilibrium of the original game if and only if it is an equilibrium of this transformed game.

\section{A.2 Proofs}

Lemma 4 For any strategy $\sigma_{i}$ of any agent $i$, there exists $x_{0} \in[0,1]$ such that

$$
\frac{w\left(x_{0}, \sigma_{i}, 0\right)}{w\left(x_{0}, \sigma_{i}, 1\right)}=\frac{w\left(\varnothing, \sigma_{i}, 0\right)}{w\left(\varnothing, \sigma_{i}, 1\right)}
$$

Consequently, for any strategy profile $\hat{\sigma}$ in which the moderator acts optimally and agent $i$ 's strategy is $\sigma_{i}$, we have $u_{i}\left(x_{0} ; \hat{\sigma}\right)=u_{i}(\varnothing ; \hat{\sigma})$.

Proof. By the definition of $w$,

$$
\begin{aligned}
\frac{w\left(\varnothing, \sigma_{i}, 0\right)}{w\left(\varnothing, \sigma_{i}, 1\right)} & =\frac{h\left(\sigma_{i}, 0\right)}{h\left(\sigma_{i}, 1\right)} \\
& =\frac{\int_{0}^{1}\left(1-\sigma_{i}(x)\right) f_{0}(x) d x}{\int_{0}^{1}\left(1-\sigma_{i}(x)\right) f_{1}(x) d x} .
\end{aligned}
$$


Let $M=\sup _{x \in[0,1]} f_{0}(x) / f_{1}(x)$ and $m=\inf _{x \in[0,1]} f_{0}(x) / f_{1}(x)$, so that $f_{0}(x)<M f_{1}(x)$ and $f_{0}(x)>m f_{1}(x)$ for all $x \in(0,1)$. Then

$$
m<\frac{\int_{0}^{1}\left(1-\sigma_{i}(x)\right) f_{0}(x) d x}{\int_{0}^{1}\left(1-\sigma_{i}(x)\right) f_{1}(x) d x}<M .
$$

So by the continuity of $f_{0}$ and $f_{1}$, there exists $x_{0}$ such that

$$
\frac{f_{0}\left(x_{0}\right)}{f_{1}\left(x_{0}\right)}=\frac{\int_{0}^{1}\left(1-\sigma_{i}(x)\right) f_{0}(x) d x}{\int_{0}^{1}\left(1-\sigma_{i}(x)\right) f_{1}(x) d x},
$$

which completes the argument since

$$
\frac{w\left(x, \sigma_{i}, 0\right)}{w\left(x, \sigma_{i}, 1\right)}=\frac{f_{0}(x)}{f_{1}(x)}
$$

for all $x \in(0,1)$.

For the second claim, the action of type $x_{0}$ does not affect the moderator's choice unless the ratio on the left-hand side of (1) is equal to 1 . In that case, the expected payoff is independent of the moderator's choice.

Proof of Proposition 1. Let $\sigma$ be a symmetric strategy profile in which the moderator acts optimally. For any type $x_{i}$ of agent $i$, define the incentive to reveal for type $x_{i}, I_{i}\left(x_{i}\right)$, to be the difference between $i$ 's expected payoffs from revealing and not revealing her signal. That is,

$$
\begin{aligned}
I_{i}\left(x_{i}\right)=P\left(0 \mid x_{i}\right)\left(\psi\left(0, x_{i}, 0 ; \sigma\right)-\psi\right. & (0, \varnothing, 0 ; \sigma)) \\
& +P\left(1 \mid x_{i}\right)\left(\psi\left(1, x_{i}, 1 ; \sigma\right)-\psi(1, \varnothing, 1 ; \sigma)\right)-c .
\end{aligned}
$$

Let $x_{0}$ be as in Lemma 4 for $\sigma_{i}$. The claims in the first two sentences of the result follow if, for each agent $i, I_{i}$ is continuous, decreasing on $\left[0, x_{0}\right)$, and increasing on $\left(x_{0}, 1\right]$.

From Lemma 4 we have $\psi(0, \varnothing, 0 ; \sigma)=\psi\left(0, x_{0}, 0 ; \sigma\right)$ and $\psi(0, \varnothing, 1 ; \sigma)=$ $\psi\left(0, x_{0}, 1 ; \sigma\right)$, so

$$
\begin{aligned}
I_{i}\left(x_{i}\right)=P\left(0 \mid x_{i}\right)\left(\psi\left(0, x_{i}, 0 ; \sigma\right)-\psi(0,\right. & \left.\left.x_{0}, 0 ; \sigma\right)\right) \\
& +P\left(1 \mid x_{i}\right)\left(\psi\left(1, x_{i}, 1 ; \sigma\right)-\psi\left(1, x_{0}, 1 ; \sigma\right)\right)-c .
\end{aligned}
$$

Since agent $i$ has the same preference over the options as does the moderator, her expected payoff from the moderator's action if she reveals her signal $x_{i}$ and 
the moderator acts as if she had revealed $x_{i}^{\prime} \neq x_{i}$ is no higher than her expected payoff from revealing $x_{i}$. That is,

$$
\begin{aligned}
P\left(0 \mid x_{i}\right) \psi\left(0, x_{i}, 0 ; \sigma\right)+P\left(1 \mid x_{i}\right) \psi & \left(1, x_{i}, 1 ; \sigma\right) \\
& \geq P\left(0 \mid x_{i}\right) \psi\left(0, x_{i}^{\prime}, 0 ; \sigma\right)+P\left(1 \mid x_{i}\right) \psi\left(1, x_{i}^{\prime}, 1 ; \sigma\right)
\end{aligned}
$$

for every $x_{i}$. It follows that

$$
\begin{aligned}
I_{i}\left(x_{i}\right) \geq P\left(0 \mid x_{i}\right)\left(\psi\left(0, x_{i}^{\prime}, 0 ; \sigma\right)-\psi\left(0, x_{0}, 0 ; \sigma\right)\right) & \\
& +P\left(1 \mid x_{i}\right)\left(\psi\left(1, x_{i}^{\prime}, 1 ; \sigma\right)-\psi\left(1, x_{0}, 1 ; \sigma\right)\right)-c \\
=I_{i}\left(x_{i}^{\prime}\right)+\left(P\left(0 \mid x_{i}\right)-P\left(0 \mid x_{i}^{\prime}\right)\right)\left(\psi\left(0, x_{i}^{\prime}, 0 ; \sigma\right)-\psi\left(0, x_{0}, 0 ; \sigma\right)\right. & \left.-\psi\left(1, x_{i}^{\prime}, 1 ; \sigma\right)+\psi\left(1, x_{0}, 1 ; \sigma\right)\right)
\end{aligned}
$$

for every $x_{i}^{\prime}$. Now suppose $x_{i}<x_{i}^{\prime}<x_{0}$. Since $x_{i}^{\prime}<x_{0}, \psi\left(0, x_{i}^{\prime}, 0 ; \sigma\right)>\psi\left(0, x_{0}, 0 ; \sigma\right)$ and $\psi\left(1, x_{0}, 1 ; \sigma\right)>\psi\left(1, x_{i}^{\prime}, 1 ; \sigma\right)$. Since $x_{i}<x_{i}^{\prime}, P\left(0 \mid x_{i}\right)>P\left(0 \mid x_{i}^{\prime}\right)$. Together with (8), these inequalities imply that $I_{i}\left(x_{i}\right)>I_{i}\left(x_{i}^{\prime}\right)$. Therefore, $I_{i}$ is decreasing on $\left[0, x_{0}\right]$. A symmetric argument shows that it is increasing on $\left[x_{0}, 1\right]$.

Finally, we show that $I_{i}$ is continuous. We have

$$
\begin{aligned}
I_{i}\left(x_{i}\right)=P\left(0 \mid x_{i}\right) \psi\left(0, x_{i}, 0 ; \sigma\right) & +P\left(1 \mid x_{i}\right) \psi\left(1, x_{i}, 1 ; \sigma\right) \\
& -P\left(0 \mid x_{i}\right) \psi(0, \varnothing, 0 ; \sigma)-P\left(1 \mid x_{i}\right) \psi(1, \varnothing, 1 ; \sigma)-c .
\end{aligned}
$$

The third and fourth terms are continuous in $x_{i}$ by the continuity of $f_{0}$ and $f_{1}$. The sum of the first two terms is the moderator's expected payoff conditional on agent $i$ 's having signal $x_{i}$ and revealing it. The moderator's expected payoff conditional on reaching the information set $a$ is $\max \left\{\beta_{\sigma}(0 \mid a), 1-\beta_{\sigma}(0 \mid a)\right\}$, where $\beta_{\sigma}(0 \mid a)$ is, as before, the probability assigned to state 0 by the moderator's belief at her information set $a$. The probability $\beta_{\sigma}(0 \mid a)$ is continuous in $a_{i}$ for $a_{i} \in[0,1]$ for any given values of $a_{j}$ for $j \neq i$, so that taking the expectation over the other agents' actions conditional on $a_{i}$ shows that the moderator's expected payoff conditional on agent $i$ 's having signal $a_{i}$ and revealing it is continuous in $a_{i}$, so that $I_{i}$ is continuous.

Lemma 5 Consider a symmetric strategy profile in which the common strategy of the agents is a threshold strategy with thresholds $\underline{x}$ and $\bar{x}$ and the moderator acts optimally. The probability that the option chosen by the moderator is equal to the state is nondecreasing in $\underline{x}$ and nonincreasing in $\bar{x}$.

Proof. Increasing $\underline{x}$ or decreasing $\bar{x}$ makes the signals provided to the moderator more informative in the sense of Blackwell. Therefore such a change cannot 
reduce her expected payoff, which is the probability that she chooses the option equal to the state.

Lemma 6 For any $\varepsilon>0$ and $\delta>0$ there exists $N$ such that for any $n>N$ and threshold strategy $\hat{\sigma}$ such that $\underline{x} \geq \varepsilon$ or $\bar{x} \leq 1-\varepsilon$ (or both), where $\underline{x}$ and $\bar{x}$ are the thresholds, if every agent uses the strategy $\hat{\sigma}$ and the moderator acts optimally then the moderator's expected payoff exceeds $1-\delta$ in each state.

Proof. First suppose that all agents use the strategy $\hat{\sigma}$ for which $\underline{x}=\varepsilon$ and $\bar{x}=1$. For any information set $\left(a_{1}, \ldots, a_{n}\right)$ of the moderator, let

$$
\rho\left(a_{1}, \ldots, a_{n}\right)=\frac{q}{1-q} \prod_{i=1}^{n} \frac{w\left(a_{i}, \hat{\sigma}, 0\right)}{w\left(a_{i}, \hat{\sigma}, 1\right)},
$$

and recall (see (1)) that the moderator chooses option 0 whenever $\rho\left(a_{1}, \ldots, a_{n}\right)>$ 1 and option 1 whenever $\rho\left(a_{1}, \ldots, a_{n}\right)<1$.

Note that for every agent $i$

$$
\begin{aligned}
E\left[\frac{w\left(a_{i}, \hat{\sigma}, 0\right)}{w\left(a_{i}, \hat{\sigma}, 1\right)} \mid s=1\right] & =\operatorname{Pr}_{\hat{\sigma}}\left(a_{i}=\varnothing \mid s=1\right) \frac{h(\hat{\sigma}, 0)}{h(\hat{\sigma}, 1)}+\int_{x \in[0, \varepsilon]} \frac{f_{0}(x)}{f_{1}(x)} d F_{1}(x) \\
& =\operatorname{Pr}_{\hat{\sigma}}\left(a_{i}=\varnothing \mid s=1\right) \frac{\operatorname{Pr}_{\hat{\sigma}}\left(a_{i}=\varnothing \mid s=0\right)}{\operatorname{Pr}_{\hat{\sigma}}\left(a_{i}=\varnothing \mid s=1\right)}+\int_{x \in[0, \varepsilon]} f_{0}(x) d x \\
& =\operatorname{Pr}\left(x_{i} \in[\varepsilon, 1] \mid s=0\right)+\operatorname{Pr}\left(x_{i} \in[0, \varepsilon] \mid s=0\right) \\
& =1 .
\end{aligned}
$$

Thus, conditional on $s=1,\left(\rho\left(a_{1}, \ldots, a_{n}\right)\right)_{n=1}^{\infty}$ is a nonnegative martingale, since actions are (conditionally) independent across agents. Therefore by Shiryaev (1996, Corollary 3, p. 509), $\rho\left(a_{1}, \ldots, a_{n}\right)$ converges almost surely as $n \rightarrow \infty$. We have $\operatorname{Pr}_{\hat{\sigma}}\left(a_{i} \neq \varnothing \mid s=1\right)>0$ (since $\underline{x}>0$ ) and $f_{0}(x) \neq f_{1}(x)$ for almost all $x$, so $\operatorname{Pr}\left(w_{i}\left(a_{i}, \hat{\sigma}, 0\right) \neq w_{i}\left(a_{i}, \hat{\sigma}, 1\right) \mid s=1\right)>0$. Hence the random variable to which $\rho\left(a_{1}, \ldots, a_{n}\right)$ converges is equal to 0 almost surely. Symmetrically, $\rho\left(a_{1}, \ldots, a_{n}\right)$ diverges to $\infty$ almost surely conditional on $s=0$. Thus given any $\delta>0$, there exists a number $\underline{N}(\varepsilon, \delta)$ such that for any $n>\underline{N}(\varepsilon, \delta)$,

$$
\begin{array}{r}
\operatorname{Pr}\left(\rho\left(a_{1}, \ldots, a_{n}\right)>1 \mid s=0\right)>1-\delta \\
\text { and } \operatorname{Pr}\left(\rho\left(a_{1}, \ldots, a_{n}\right)<1 \mid s=1\right)>1-\delta .
\end{array}
$$

If all agents use the strategy with $\underline{x}=0$ and $\bar{x}=1-\varepsilon$, a symmetric argument shows that there exists $\bar{N}(\varepsilon, \delta)$ for which (9) holds whenever $n>\bar{N}(\varepsilon, \delta)$. Letting 
$N(\varepsilon, \delta)=\max \{\underline{N}(\varepsilon, \delta), \bar{N}(\varepsilon, \delta)\}$, Lemma 5 implies that (9) holds for all $n>N(\varepsilon, \delta)$ whenever all agents use a common threshold strategy with either $\underline{x} \geq \varepsilon$ or $\bar{x} \leq$ $1-\varepsilon$ and the moderator acts optimally.

Lemma 7 There exists $\bar{\eta}>0$ such that, for any $n$, any symmetric equilibrium $\sigma$, any agent $i$, and each state $s$, the probability that $i$ does not reveal a signal, $\operatorname{Pr}\left(\left\{x_{i}:\right.\right.$ $\left.\left.\sigma_{i}\left(x_{i}\right)=0\right\} \mid s\right)$, is at least $\bar{\eta}$.

Proof. Note that the function $\tilde{q}:[0,1]^{2} \rightarrow[0,1]$ defined by

$$
\tilde{q}(p, x)=\frac{p f_{0}(x)}{p f_{0}(x)+(1-p) f_{1}(x)}
$$

is continuous. Moreover, since its domain is compact, the continuity is uniform. In particular, there exists $\gamma>0$ such that $\left|\tilde{q}(p, x)-\tilde{q}\left(p, x^{\prime}\right)\right|<c / 2$ for all $p$ whenever $\left|x-x^{\prime}\right|<\gamma$.

Let $\underline{x}$ and $\bar{x}$ be the thresholds for a symmetric equilibrium strategy (for given $n$ ). Assume that $\underline{x}>0$. (The case $\bar{x}<1$ is similar.) Suppose that all agents other than $i$ follow the equilibrium strategy. If agent $i$ 's signal is $\underline{x}$ and she reveals it, the posterior probability the moderator assigns to state 0 is at least as high as if agent $i$ does not reveal her signal. Revealing $\underline{x}$ therefore affects the payoff from the moderator's choice only if it switches the posterior belief from less than $\frac{1}{2}$ to greater than $\frac{1}{2}$, in which case it provides an expected gain of $q^{\prime}-\left(1-q^{\prime}\right)=2 q^{\prime}-1$, where $q^{\prime}$ is the posterior belief that the state is 0 when $i$ reveals $\underline{x}$. For any list $a_{-i}$ of the actions of the players other than $i$, let $q\left(a_{-i}\right)$ be the posterior probability of state 0 given $a_{-i}$ and let $x_{0}$ be the signal given by Lemma 4 for the strategy $\sigma_{i}$. Then the condition that type $\underline{x}$ be indifferent between revealing her signal and not revealing it is

$$
\begin{aligned}
& \operatorname{Pr}_{\sigma}\left(\left\{a_{-i} \mid \tilde{q}\left(q\left(a_{-i}\right), \underline{x}\right)>\frac{1}{2} \text { and } \tilde{q}\left(q\left(a_{-i}\right), x_{0}\right)<\frac{1}{2}\right\}\right) \times \\
& E\left[2 \tilde{q}\left(q\left(a_{-i}\right), \underline{x}\right)-1 \mid \tilde{q}\left(q\left(a_{-i}, \underline{x}\right)>\frac{1}{2} \text { and } \tilde{q}\left(q\left(a_{-i}\right), x_{0}\right)<\frac{1}{2}\right]=c .\right.
\end{aligned}
$$

By the argument in the first paragraph of the proof, if $\left|\underline{x}-x_{0}\right|<\gamma$ then $\tilde{q}(p, \underline{x})<$ $(1+c) / 2$ whenever $\tilde{q}\left(p, x_{0}\right)<\frac{1}{2}$, in which case the left-hand side of (10) is less than $c$. Hence $\left|\underline{x}-x_{0}\right| \geq \gamma$, and thus for $s=0,1$ the probability that agent $i$ does not reveal a signal is

$$
\begin{aligned}
\operatorname{Pr}\left(\left\{x_{i}: \sigma_{i}\left(x_{i}\right)=0\right\} \mid s\right) & =F_{s}(\bar{x})-F_{s}(\underline{x}) \\
& \geq F_{s}\left(x_{0}\right)-F_{s}(\underline{x}) \\
& \geq \min _{x \in[0,1-\gamma]} F_{s}(x+\gamma)-F_{s}(x),
\end{aligned}
$$


where the second line follows from Proposition 1, which says that $x_{0} \in(\underline{x}, \bar{x})$.

Taking $\bar{\eta}=\min _{s \in\{0,1\}} \min _{x \in[0,1-\gamma]} F_{s}(x+\gamma)-F_{s}(x)$ gives the result.

Proof of Lemma 1. Choose $\delta<c \bar{\eta}$, where $\bar{\eta}$ comes from Lemma 7. Given $\varepsilon>0$, let $N$ be the number given by Lemma 6 . Then for any $n>N$ and any threshold strategy $\sigma$ with $\underline{x} \geq \varepsilon$ or $\bar{x} \leq 1-\varepsilon$, we have

$$
\begin{aligned}
& 1-\delta< \operatorname{Pr}\left(\left\{x_{i}: \sigma\left(x_{i}\right)=0\right\} \mid 0\right) \psi(0, \varnothing, 0 ; \sigma)+\int_{\left.x_{i} \in[0,1] \backslash \underline{x}, \bar{x}\right)} \psi\left(0, x_{i}, 0 ; \sigma\right) d F_{0}\left(x_{i}\right) \\
&=\bar{\eta} \psi(0, \varnothing, 0 ; \sigma)+\left(\operatorname{Pr}\left(\left\{x_{i}: \sigma\left(x_{i}\right)=\varnothing\right\} \mid 0\right)-\bar{\eta}\right) \psi(0, \varnothing, 0 ; \sigma)+\int_{x_{i} \in[0,1] \backslash(\underline{x}, \bar{x})} \psi\left(0, x_{i}, 0 ; \sigma\right) d F_{0}\left(x_{i}\right) \\
& \leq \bar{\eta} \psi(0, \varnothing, 0 ; \sigma)+\operatorname{Pr}\left(\left\{x_{i}: \sigma\left(x_{i}\right)=\varnothing\right\} \mid 0\right)-\bar{\eta}+1-\operatorname{Pr}\left(\left\{x_{i}: \sigma\left(x_{i}\right)=\varnothing\right\} \mid 0\right) \\
& \leq \bar{\eta} \psi(0, \varnothing, 0 ; \sigma)+1-\bar{\eta} .
\end{aligned}
$$

Thus $\psi(0, \varnothing, 0 ; \sigma)>1-\delta / \bar{\eta}$. Similarly, $\psi(1, \varnothing, 1 ; \sigma)>1-\delta / \bar{\eta}$. Therefore, for any type, the expected payoff from nonrevelation is greater than $1-\delta / \bar{\eta}$, which, by the choice of $\delta$, is greater than $1-c$, the highest possible payoff from revelation. Therefore the strategy profile in which all agents use the thresholds $\underline{x}$ and $\bar{x}$ is not an equilibrium.

Proof of Lemma 2. Suppose $\underline{x}>0$. The option chosen by the moderator when all agents but $i$ follow the strategy profile $\sigma$ and agent $i$ does not reveal her signal differs from the option the moderator chooses if all agents use $\sigma$ only if agent $i$ 's signal is in $[0, \underline{x}) \cup(\bar{x}, 1]$. For any given signal $x_{i}$ the difference between the probabilities that the moderator chooses option 0 in the two cases is at most 1 , so that the difference between the probabilities that the moderator chooses option 0 in the two cases when the state is 0 is at $\operatorname{most} \operatorname{Pr}\left(x_{i} \in[0, \underline{x}) \cup(\bar{x}, 1] \mid s=0\right)$. Denoting the probability that the moderator chooses option $a$ when the state is $s$ and all players use $\sigma$ by $\Psi(a, s ; \sigma)$, we thus have

$$
|\Psi(0,0 ; \sigma)-\psi(0, \varnothing, 0 ; \sigma)| \leq \operatorname{Pr}\left(x_{i} \in[0, \underline{x}) \cup(\bar{x}, 1] \mid s=0\right) .
$$

By Lemma 1, for $n$ sufficiently large we have $\operatorname{Pr}\left(x_{i} \in[0, \underline{x}) \cup(\bar{x}, 1] \mid s=0\right)<\varepsilon$, so that

$$
|\Psi(0,0 ; \sigma)-\psi(0, \varnothing, 0 ; \sigma)|<\varepsilon
$$


and hence

$$
P(0 \mid \underline{x}) \Psi(0,0 ; \sigma)<P(0 \mid \underline{x})(\psi(0, \varnothing, 0 ; \sigma)+\varepsilon) .
$$

Now, given $\underline{x}>0$, by Proposition 1 the indifference condition (3) is satisfied. Substituting for $\psi(0, \varnothing, 0 ; \sigma)$ from (3) leads to

$$
\begin{aligned}
P(0 \mid \underline{x}) \Psi(0,0 ; \sigma)<P(0 \mid \underline{x})(\psi(0, \underline{x}, 0 ; \sigma)+\varepsilon) \\
+P(1 \mid \underline{x})(\psi(1, \underline{x}, 1 ; \sigma)-\psi(1, \varnothing, 1 ; \sigma))-c .
\end{aligned}
$$

Let $x_{0}$ be the signal given in Lemma 4 for the common strategy of each agent in $\sigma$. Then $\psi(1, \underline{x}, 1 ; \sigma)-\psi(1, \varnothing, 1 ; \sigma)=\psi(1, \underline{x}, 1 ; \sigma)-\psi\left(1, x_{0}, 1 ; \sigma\right)<0$ since $\underline{x}<x_{0}$ by Proposition 1 , so that revealing the signal $\underline{x}$ increases the probability that the moderator chooses option 0 and thus decreases the probability that she chooses option 1. Dividing both sides of (12) by $P(0 \mid \underline{x})$ and omitting the negative term, we have

$$
\begin{aligned}
\Psi(0,0 ; \sigma) & <\psi(0, \underline{x}, 0 ; \sigma)+\varepsilon-c / P(0 \mid \underline{x}) \\
& <1-c+\varepsilon
\end{aligned}
$$

as needed.

A symmetric argument shows that if $\bar{x}<1$ then the probability that the moderator chooses option 1 in state 1 is at most $1-c+\varepsilon$.

Lemma 8 Let $\hat{q}=\min (q, 1-q)$. Then for any $\delta \in(0, c \hat{q})$, there exists $N$ such that for any $n>N$, in any equilibrium with thresholds $\underline{x}$ and $\bar{x}$ in which either $\underline{x}>0$ or $\bar{x}<1$, the moderator's expected payoff is at most $1-\delta$.

Proof. Suppose $\underline{x}>0$. Let $N$ be the number given in Lemma 2 for $\varepsilon=c-\delta / \hat{q}>0$, so that in a symmetric equilibrium the probability of the moderator's choosing option 0 in state 0 is at most $1-c / P(0 \mid 0)+\varepsilon \leq 1-c+\varepsilon=1-\delta / \hat{q}$. Thus the moderator's expected payoff in the equilibrium is at most

$$
q(1-\delta / \hat{q})+(1-q) \cdot 1=1-\delta q / \hat{q} \leq 1-\delta .
$$

A symmetric argument leads to the same conclusion if $\bar{x}<1$.

Lemma 9 In any equilibrium with thresholds $\underline{x}$ and $\bar{x}$, the moderator's expected payoff is at least $1-4 D / n M^{2}$, where $M=\max \left\{F_{0}(\underline{x})-F_{1}(\underline{x}), F_{0}(\bar{x})-F_{1}(\bar{x})\right\}$ and $D=\max \left\{F_{0}(\underline{x}), F_{1}(\underline{x}), 1-F_{0}(\bar{x}), 1-F_{1}(\bar{x})\right\}$. 
Proof. Suppose that $F_{0}(\underline{x})-F_{1}(\underline{x})>F_{0}(\bar{x})-F_{1}(\bar{x})$. Denote by $Y$ be the (random) number of agents $i$ for whom $x_{i}<\underline{x}$, so that in state $i$ the mean of $Y$ is $n F_{i}(\underline{x})$. Suppose the moderator selects option 0 if and only if $Y>n\left(F_{0}(\underline{x})+F_{1}(\underline{x})\right) / 2$. Then in each state the moderator selects the wrong option if $Y$ differs from its mean by more than $\Delta=n\left(F_{0}(\underline{x})-F_{1}(\underline{x})\right) / 2 \geq n M / 2$. Now, the distribution of $Y$ in state $i$ is $\operatorname{Binomial}\left(n, F_{i}(\underline{x})\right)$, with variance $v=n F_{i}(\underline{x})\left(1-F_{i}(\underline{x})\right) \leq n F_{i}(\underline{x}) \leq n D$. So by Chebyshev's inequality, the probability that $Y$ differs from its mean by more than $\Delta$ is at most $v / \Delta^{2} \leq(n D) /(n M / 2)^{2}=4 D / n M^{2}$. Hence the probability that this strategy of the moderator chooses the right option is at least $1-4 D / n M^{2}$. Thus the probability that the moderator's optimal strategy chooses the right option is also at least this number, so that the moderator's expected payoff in an equilibrium is at least the same number.

A symmetric argument establishes the same inequality if $F_{0}(\underline{x})-F_{1}(\underline{x}) \leq F_{0}(\bar{x})-$ $F_{1}(\bar{x})$.

Proof of Lemma 3. If $\underline{x}=0$ and $\bar{x}=1$ then the number of agents who reveal signals is zero, so the result follows. So assume $\underline{x}>0$ or $\bar{x}<1$. Choose $\alpha>1$ and $\varepsilon>0$ small enough that $f_{0}(\varepsilon) \geq \alpha f_{1}(\varepsilon)$ and $f_{1}(1-\varepsilon) \geq \alpha f_{0}(1-\varepsilon)$. Let $N$ be the number given in Lemma 1 for $\varepsilon$, so that for $n>N$ we have $\underline{x}<\varepsilon$ and $1-\bar{x}<\varepsilon$. Let $\hat{q}=\min (q, 1-q)$ and $\delta<c \hat{q} / 2$.

Combining Lemmas 8 and 9 , we have $1-4 D / n M^{2} \leq 1-\delta$, where $D$ and $M$ are given in Lemma 9 , so that $4 D / \delta M^{2} \geq n$.

Since $\underline{x} \leq \varepsilon$ and $\bar{x} \geq 1-\varepsilon$, we have $F_{0}(\underline{x}) \geq \alpha F_{1}(\underline{x})$ and $1-F_{1}(\bar{x}) \geq \alpha\left(1-F_{0}(\bar{x})\right)$. Thus

$$
\begin{gathered}
M=\max \left\{F_{0}(\underline{x})-F_{1}(\underline{x}),\left(1-F_{1}(\bar{x})\right)-\left(1-F_{0}(\bar{x})\right)\right\} \\
\geq \max \left\{F_{0}(\underline{x})-F_{0}(\underline{x}) / \alpha,\left(1-F_{1}(\bar{x})\right)-\left(1-F_{1}(\bar{x})\right) / \alpha\right\} \\
=\max \left\{(1-1 / \alpha) F_{0}(\underline{x}),(1-1 / \alpha)\left(1-F_{1}(\bar{x})\right)\right\} \\
=(1-1 / \alpha) D,
\end{gathered}
$$

where the last equality follows from the facts that $F_{1}(\underline{x})<F_{0}(\underline{x})$ (because $f_{0}(\underline{x})>$ $f_{1}(\underline{x})$ ) and $1-F_{0}(\bar{x})<1-F_{1}(\bar{x})$ (because $f_{1}(\bar{x})>f_{0}(\bar{x})$ ).

Thus $n \leq 4 D / \delta M^{2} \leq 4 \alpha^{2} / \delta(\alpha-1)^{2} D$. But then the expected number of agents who reveal their signals is at most

$$
n \max \left\{F_{0}(\underline{x})+1-F_{0}(\bar{x}), F_{1}(\underline{x})+1-F_{1}(\bar{x})\right\} \leq 2 n D \leq 8 \alpha^{2} / \delta(\alpha-1)^{2},
$$

and in particular is bounded independently of $n$. 
Proof of Proposition 2. Consider a two-sided equilibrium with thresholds $\underline{x}>0$ and $\bar{x}<1$ in which the moderator chooses option 0 if no signal is revealed. Let $X$ denote the number of agents with signals at least $\bar{x}$. Then

$$
\frac{E[X \mid s=0]}{E[X \mid s=1]}=\frac{n\left(1-F_{0}(\bar{x})\right)}{n\left(1-F_{1}(\bar{x})\right)}=\frac{1-F_{0}(\bar{x})}{1-F_{1}(\bar{x})}
$$

and

$$
\lim _{\bar{x} \rightarrow 1} \frac{1-F_{0}(\bar{x})}{1-F_{1}(\bar{x})}=\lim _{\bar{x} \rightarrow 1} \frac{f_{0}(\bar{x})}{f_{1}(\bar{x})}=0 .
$$

By Lemma $1, \bar{x}$ converges to 1 as the number of agents increases, so for any sequence of equilibria, one for each $n, E[X \mid s=0] / E[X \mid s=1]$ converges to 0 as $n$ increases. By Lemma 3, $E[X \mid s=1]$ is bounded, so $E[X \mid s=0]$ converges to 0 and hence $\operatorname{Pr}(X=0 \mid s=0)$ converges to 1 . Since, by assumption, the moderator chooses option 0 if no agent reveals her signal, she chooses option 0 also whenever $X=0$ (in which case all revealed signals are at most $\underline{x}$ ). Therefore, the probability that the moderator chooses option 0 in state 0 converges to 1 , contradicting Lemma 2.

For the second statement, the posterior probability of state 0 if one agent reveals the signal $\bar{x}$ and no one else reveals a signal is

$$
\frac{q f_{0}(\bar{x}) F_{0}(\bar{x})^{n-1}}{q f_{0}(\bar{x}) F_{0}(\bar{x})^{n-1}+(1-q) f_{1}(\bar{x}) F_{1}(\bar{x})^{n-1}}=\frac{1}{1+\frac{1-q}{q} \frac{F_{1}(\bar{x})^{n-1}}{F_{0}(\bar{x})^{n-1}} \frac{f_{1}(\bar{x})}{f_{0}(\bar{x})}} .
$$

By Lemma 1 and the fact that $f_{0}(1)=0, f_{1}(\bar{x}) / f_{0}(\bar{x})$ increases without bound as $n$ increases. We claim that it suffices to show that $F_{1}(\bar{x})^{n-1}$ is bounded away from 0 as $n$ increases. The reason is that in this case the posterior (13) approaches 0 , so that for sufficiently large $n$ the moderator chooses option 1 whenever at least one agent reveals a signal of at least $\bar{x}$.

Let $p(n)=1-F_{1}(\bar{x}(n))$, the probability in state 1 that a signal is revealed in the equilibrium when there are $n$ agents. By Lemma 3, $n p(n)$, the expected number of signals revealed, remains bounded as $n$ increases without bound. Denote the bound by $B$. Then $p(n)<B / n$ for all $n$, so that $(1-p(n))^{n}>(1-B / n)^{n}$ for all $n$. Given that $\lim _{n \rightarrow \infty}(1-B / n)^{n}=e^{-B}>0$, we conclude that $\lim _{n \rightarrow \infty}(1-p(n))^{n}=$ $\lim _{n \rightarrow \infty} F_{1}(\bar{x}(n))^{n}>e^{-B}>0$. Hence $F_{1}(\bar{x}(n))^{n-1}$ is also bounded away from 0 as $n$ increases without bound.

Proof of Proposition 3. In such an equilibrium $\sigma$, (4) holds. By Lemma 1, $P(0 \mid \bar{x})$ approaches 0 and $P(1 \mid \bar{x})$ approaches 1 as $n$ increases. By Proposition 2, for large enough $n$ we have $z_{1}(\bar{x}, n)<\bar{x}$, so that $\psi(1, \bar{x}, 1 ; \sigma)=1$. Therefore $\psi(1, \varnothing, 1 ; \sigma)$ 
approaches $1-c$. By the analogue of (11) for state $1, \psi(1, \varnothing, 1 ; \sigma)$ approaches $\Psi(1,1 ; \sigma)$ a $n$ increases, as needed.

By Proposition 2, the expected number of agents who reveal signals in state 0 approaches 0 as $n$ increases. Let $\pi=1-F_{1}(\bar{x})$, the probability that in state 1 an agent reveals her signal. Then when the number of agents is large, the expected payoff of an agent in state 1 is the probability that at least one signal is revealed, namely $1-(1-\pi)^{n}$. By the previous paragraph, this expected payoff approaches $1-c$, so that for large $n, 1-(1-\pi)^{n} \approx 1-c$. Thus the expected number $n \pi$ of agents who reveal signals in state 1 satisfies

$$
\begin{aligned}
n \pi & \approx n\left(1-c^{1 / n}\right) \\
& \approx n\left(1-e^{(\ln c) / n}\right) \\
& \approx n\left(1-\left(1+\frac{1}{n} \ln c\right)\right) \\
& \approx-\ln c,
\end{aligned}
$$

so that the expected number of agents who reveal signals is $-(1-q) \ln c$, completing the argument.

Lemma 10 A strategy profile that maximizes the average payoffof the agents among those in which the moderator acts optimally for the game with cost $c$ is an equilibrium of the game in which each agent's revelation cost is $c / n$.

Proof. For a given strategy profile, the agents' average payoff is

$$
\operatorname{Pr}(\text { correct decision })-\sum_{i=1}^{n} \operatorname{Pr}(i \text { reveals her signal }) c / n \text {. }
$$

Consider a strategy profile $\sigma$ that maximizes this average payoff among those in which the moderator acts optimally. Suppose that $\sigma$ is not an equilibrium of the game with cost $c / n$. Then some agent $i$ in that game has a profitable deviation, say to the strategy $\sigma_{i}^{\prime}$. Consider the game in which each agent's revelation cost is $c / n$. In that game, relative to $\sigma$, the strategy profile $\left(\sigma_{-i}, \sigma_{i}^{\prime}\right)$ increases the payoff of agent $i$, namely

$$
\operatorname{Pr}(\text { correct decision })-\operatorname{Pr}(i \text { reveals her signal }) c / n,
$$

and does not affect the probability that any other agent reveals her signal. Therefore $\left(\sigma_{-i}, \sigma_{i}^{\prime}\right)$ yields higher average payoff (with cost $c$ ) than does $\sigma$, contradicting our assumption that $\sigma$ maximizes the agents' average payoff. 
Proof of Proposition 4. The transfer scheme defined in the result satisfies budget balance since

$$
\sum_{i=1}^{n} t_{i}(a)=k(a)(1-k(a) / n) c-(n-k(a)) k(a) c / n=0 .
$$

In addition, when $k$ signals are revealed, an agent who switches from not revealing her signal to revealing it goes from paying a tax of $k c / n$ to receiving a subsidy of $(1-(k+1) / n) c$, causing her transfer to increase by $(n-1) c / n$. Thus the net cost of revealing a signal is $c / n$, so that the result follows from Lemma 10.

Proof of Proposition 5. Let $\sigma$ be the strategy profile in our original game in which the agents follow the equilibrium of $G(d)$ and the moderator's strategy is $d$.

If, according to $\sigma$, no type of any agent reveals her signal, then the expected payoff of each type of every agent is at $\operatorname{most} \max \{q, 1-q\}$.

Now suppose that according to $\sigma$, type $x_{i}$ of agent $i$ reveals her signal. Then the optimality of revelation for type $x_{i}$ of agent $i$ means that

$$
\begin{aligned}
P\left(0 \mid x_{i}\right) \psi_{i}\left(0, x_{i}, 0 ; \sigma\right)+P\left(1 \mid x_{i}\right) \psi_{i}\left(1, x_{i}, 1 ; \sigma\right)-c & \\
& \geq P\left(0 \mid x_{i}\right) \psi_{i}(0, \varnothing, 0 ; \sigma)+P\left(1 \mid x_{i}\right) \psi_{i}(1, \varnothing, 1 ; \sigma) .
\end{aligned}
$$

The left-hand side of (14) is at most $1-c$, so that either $\psi_{i}(0, \varnothing, 0 ; \sigma)$ or $\psi_{i}(1, \varnothing, 1 ; \sigma)$ (or both) is at most $1-c$. Suppose that $\psi_{i}(0, \varnothing, 0 ; \sigma) \leq 1-c$. Then if type $y_{i}$ of agent $i$ does not reveal her signal, her expected payoff is at most $P\left(0 \mid y_{i}\right)(1-c)+$ $P\left(1 \mid y_{i}\right)$, so that the (ex ante) expected payoff of agent $i$ is at most

$$
\begin{aligned}
E_{x_{i}}\left[\max \left\{1-c, P\left(0 \mid x_{i}\right)(1-c)+P\left(1 \mid x_{i}\right)\right\}\right] & =E_{x_{i}}\left[P\left(0 \mid x_{i}\right)(1-c)+P\left(1 \mid x_{i}\right)\right] \\
& =q(1-c)+1-q \\
& =\bar{\pi},
\end{aligned}
$$

where the third line follows from the Law of Iterated Expectations. A symmetric argument applies if $\psi_{i}(1, \varnothing, 1 ; \sigma) \leq 1-c$.

\section{References}

Battaglini, Marco (2016), "Public protests and policy making." Unpublished paper. [6]

Feddersen, Timothy J. and Wolfgang Pesendorfer (1996), "The swing voter's curse.” American Economic Review, 86, 408-424. [6] 
Feddersen, Timothy J. and Wolfgang Pesendorfer (1997), "Voting behavior and information aggregation in elections with private information." Econometrica, 65, 1029-1058. [6]

Jovanovic, Boyan (1982), “Truthful disclosure of information.” Bell Journal of Economics, 13, 36-44. [6]

Kartik, Navin, Wing Suen, and Frances Xu (2014), "Multi-sender disclosure of verifiable information.” Unpublished paper. [6]

Krishna, Vijay and John Morgan (2012), "Voluntary voting: costs and benefits." Journal of Economic Theory, 147, 2083-2123. [6]

Lohmann, Susanne (1993), "A signaling model of informative and manipulative political action.” American Political Science Review, 87, 319-333. [6]

Lohmann, Susanne (1994), "Information aggregation through costly political action.” American Economic Review, 84, 518-530. [6]

McMurray, Joseph C. (2013), "Aggregating information by voting: the wisdom of the experts versus the wisdom of the masses." Review of Economic Studies, 80, 277-312. [6]

Osborne, Martin J., Jeffrey S. Rosenthal, and Matthew A. Turner (2000), "Meetings with costly participation.” American Economic Review, 90, 927-943. [6]

Shiryaev, Albert N. (1996), Probability, second edition. Springer, New York. [25]

Verrecchia, Robert E. (1983), “Discretionary disclosure.” Journal of Accounting and Economics, 5, 179-194. [6] 